PROCEEDINGS OF THE

AMERICAN MATHEMATICAL SOCIETY

Volume 131, Number 8 , Pages 2549-255

S 0002-9939(02)06889-2

Article electronically published on November 27, 2002

\title{
ON SCHWARZ TYPE INEQUALITIES
}

\author{
K. TANAHASHI, A. UCHIYAMA, AND M. UCHIYAMA
}

(Communicated by Joseph A. Ball)

\begin{abstract}
We show Schwarz type inequalities and consider their converses. A continuous function $f:[0, \infty) \rightarrow[0, \infty)$ is said to be semi-operator monotone on $(a, b)$ if $\left\{f\left(t^{\frac{1}{2}}\right)\right\}^{2}$ is operator monotone on $\left(a^{2}, b^{2}\right)$. Let $T$ be a bounded linear operator on a complex Hilbert space $\mathcal{H}$ and $T=U|T|$ be the polar decomposition of $T$. Let $0 \leq A, B \in B(\mathcal{H})$ and $\|T x\| \leq\|A x\|,\left\|T^{*} y\right\| \leq\|B y\|$ for $x, y \in \mathcal{H}$. (1) If a non-zero function $f$ is semi-operator monotone on $(0, \infty)$, then $|\langle T x, y\rangle| \leq\|f(A) x\|\|g(B) y\|$ for $x, y \in \mathcal{H}$, where $g(t)=t / f(t)$. (2) If $f, g$ are semi-operator monotone on $(0, \infty)$, then $|\langle U f(|T|) g(|T|) x, y\rangle| \leq$ $\|f(A) x\|\|g(B) y\|$ for $x, y \in \mathcal{H}$. Also, we show converses of these inequalities, which imply that semi-operator monotonicity is necessary.
\end{abstract}

\section{INTRODUCTION}

Let $\mathcal{H}$ be a complex Hilbert space and $B(\mathcal{H})$ be the algebra of all bounded linear operators on $\mathcal{H}$. Furuta [3] extended the Heinz-Kato [5], 6] inequality, which is an extension of the Schwarz inequality.

Proposition 1 (Heinz-Kato-Furuta). Let $T=U|T|$ be the polar decomposition of $T \in B(\mathcal{H}), 0 \leq A, B \in B(\mathcal{H})$ and $\|T x\| \leq\|A x\|,\left\|T^{*} y\right\| \leq\|B y\|$ for $x, y \in \mathcal{H}$. Then

$$
\left|\left\langle T|T|^{\alpha+\beta-1} x, y\right\rangle\right| \leq\left\|A^{\alpha} x\right\|\left\|B^{\beta} y\right\|
$$

for $x, y \in \mathcal{H}, \alpha, \beta \in[0,1]$ with $1 \leq \alpha+\beta$.

The Heinz-Kato inequality is the case $\alpha+\beta=1$. Recently, M. Uchiyama [7] extended this result as follows.

Proposition 2 (M. Uchiyama). Let $f, g:[0, \infty) \rightarrow[0, \infty)$ be continuous operator monotone functions. Let $T=U|T|$ be the polar decomposition of $T \in B(\mathcal{H}), 0 \leq$ $A, B \in B(\mathcal{H})$ and $\|T x\| \leq\|A x\|,\left\|T^{*} y\right\| \leq\|B y\|$ for $x, y \in \mathcal{H}$. Then

$$
|\langle U f(|T|) g(|T|) x, y\rangle| \leq\|f(A) x\|\|g(B) y\| \quad \text { for } x, y \in \mathcal{H} .
$$

In this paper, we introduce semi-operator monotonicity which assures the conclusion of Proposition 2.

Definition 3. A continuous function $f:[0, \infty) \rightarrow[0, \infty)$ is said to be semi-operator monotone on $(a, b)$ if $\left\{f\left(t^{\frac{1}{2}}\right)\right\}^{2}$ is operator monotone on $\left(a^{2}, b^{2}\right)$.

Received by the editors December 17, 2001 and, in revised form, March 29, 2002.

2000 Mathematics Subject Classification. Primary 47A30, 47A63, 47B15.

Key words and phrases. Schwarz inequality, Heinz-Kato-Furuta inequality.

This research was supported by Grant-in-Aid Research No. 12640187. 
Proposition 4. Let $f:[0, \infty) \rightarrow[0, \infty)$ be a continuous function. Then $f$ is semi-operator monotone on $(a, b)$ if and only if $f$ has an analytic continuation for $\Pi_{1}=\left\{z \in \mathbb{C} \mid 0<\arg z<\frac{\pi}{2}\right\}$ with $f\left(\Pi_{1}\right) \subset \Pi_{1}$.

Proof. Let $f$ be semi-operator monotone on $(a, b)$. Then $g(t)=\left\{f\left(t^{\frac{1}{2}}\right)\right\}^{2}$ is operator monotone on $\left(a^{2}, b^{2}\right)$. Hence $g(t)$ has analytic continuation $g(z)=\left\{f\left(z^{\frac{1}{2}}\right)\right\}^{2}$ for $\Pi_{+}=\{z \in \mathbb{C} \mid 0<\arg z<\pi\}$ with $g\left(\Pi_{+}\right) \subset \Pi_{+}$. Hence $f\left(z^{\frac{1}{2}}\right)$ is analytic for $z \in \Pi_{+}$and $f\left(z^{\frac{1}{2}}\right) \in \Pi_{1}$. This implies that $f$ has an analytic continuation for $\Pi_{1}$ with $f\left(\Pi_{1}\right) \subset \Pi_{1}$. The converse is clear.

Every operator monotone function is semi-operator monotone. But the converse does not hold. For example, let $f(t)=\left\{\log \left(1+t^{2}\right)\right\}^{\frac{1}{2}}:[0, \infty) \rightarrow[0, \infty)$. Then $\left\{f\left(t^{\frac{1}{2}}\right)\right\}^{2}=\log (1+t)$ is operator monotone on $(0, \infty)$. But its analytic continuation $f(z)=\left\{\log \left(1+z^{2}\right)\right\}^{\frac{1}{2}}$ is singular at $z=i$. Hence $f(t)$ is not operator monotone on $(0, \infty)$. Thus the class of all semi-operator monotone functions strictly includes the class of all operator monotone functions. Also, this example shows that $g(t)=$ $\log (1+t)$ is operator monotone, but $\left\{g\left(t^{2}\right)\right\}^{\frac{1}{2}}$ is not operator monotone. Nonconstant semi-operator monotone functions are strictly increasing.

J. S. Aujla [1] and J. I. Fujii, M. Fujii [2] studied semi-operator monotone function on $(0, \infty)$ and gave several characterizations. Moreover J. I. Fujii and M. Fujii [2] studied $n$-operator monotone function on $(0, \infty)$, which generalize semi-operator monotonicity.

Since every operator monotone function $f:[0, \infty) \rightarrow[0, \infty)$ is semi-operator monotone on $(0, \infty)$, we can give a simple proof of Uchiyama's result as the following Schwarz type inequality.

Theorem 5. Let $f, g:[0, \infty) \rightarrow[0, \infty)$ be continuous functions. Let $T=U|T|$ be the polar decomposition of $T \in B(\mathcal{H}), 0 \leq A, B \in B(\mathcal{H})$ and $\|T x\| \leq\|A x\|,\left\|T^{*} y\right\| \leq$ $\|B y\|$ for $x, y \in \mathcal{H}$. Then, if $f, g$ are semi-operator monotone on $(0, \infty)$, we have

$$
|\langle U f(|T|) g(|T|) x, y\rangle| \leq\|f(A) x\|\|g(B) y\| \quad \text { for } x, y \in \mathcal{H} .
$$

Conversely, if $f, g$ satisfy the conclusion (1) and $f(t) g(t) \not \equiv 0$, then $f, g$ are semi-operator monotone on $(0, \infty)$.

Proof. Let $f, g$ be semi-operator monotone on $(0, \infty)$. Since $|T|^{2} \leq A^{2}$ and $\left|T^{*}\right|^{2} \leq$ $B^{2}$, we have $\{f(|T|)\}^{2} \leq\{f(A)\}^{2}$ and $\left\{g\left(\left|T^{*}\right|\right)\right\}^{2} \leq\{g(B)\}^{2}$. Hence

$$
\begin{aligned}
|\langle U f(|T|) g(|T|) x, y\rangle| & =\left|\left\langle g\left(\left|T^{*}\right|\right) U f(|T|) x, y\right\rangle\right| \\
& =\left|\left\langle U f(|T|) x, g\left(\left|T^{*}\right|\right) y\right\rangle\right| \\
& \leq\|f(|T|) x\|\left\|g\left(\left|T^{*}\right|\right) y\right\| \\
& \leq\|f(A) x\|\|g(B) y\| \quad \text { for } x, y \in \mathcal{H} .
\end{aligned}
$$

Conversely, take a point $a \in(0, \infty)$ such that $f(a) g(a)>0$. Let $(c, d)$ be the maximal open interval including $a$ such that $0<f(t), g(t)$ for $t \in(c, d)$. First we show that $f, g$ are semi-operator monotone on $(c, d)$. Let $\tilde{f}(t)=\left\{f\left(t^{\frac{1}{2}}\right)\right\}^{2}, \tilde{g}(t)=$ $\left\{g\left(t^{\frac{1}{2}}\right)\right\}^{2}$ for $t \in\left(c^{2}, d^{2}\right)$. Let $C \leq D, \sigma(C), \sigma(D) \subset\left(c^{2}, d^{2}\right)$. Then $C, D, g\left(C^{\frac{1}{2}}\right)$ are invertible. Let $T=C^{\frac{1}{2}}, A=D^{\frac{1}{2}}, B=C^{\frac{1}{2}}$. Then condition (1) implies

$$
\begin{aligned}
\left\|f\left(D^{\frac{1}{2}}\right) x\right\|\left\|g\left(C^{\frac{1}{2}}\right) y\right\| & \geq\left|\left\langle f\left(C^{\frac{1}{2}}\right) g\left(C^{\frac{1}{2}}\right) x, y\right\rangle\right| \\
& =\left|\left\langle f\left(C^{\frac{1}{2}}\right) x, g\left(C^{\frac{1}{2}}\right) y\right\rangle\right| \quad \text { for } x, y \in \mathcal{H} .
\end{aligned}
$$


Hence $\left\|f\left(C^{\frac{1}{2}}\right) x\right\| \leq\left\|f\left(D^{\frac{1}{2}}\right) x\right\|$ for $x \in \mathcal{H}$ and $\tilde{f}(C)=\left\{f\left(C^{\frac{1}{2}}\right)\right\}^{2} \leq\left\{f\left(D^{\frac{1}{2}}\right)\right\}^{2}=$ $\tilde{f}(D)$. Thus $f(t)$ is semi-operator monotone on $(c, d)$. Similarly we can show that $g(t)$ is semi-operator monotone on $(c, d)$.

We show $c=0$ and $d=\infty$. If $d<\infty$, then $f(d)=0$ or $g(d)=0$. But $f, g$ are positive and semi-operator monotone on $(c, d)$. Hence $0<f(d)$ and $0<g(d)$. This is a contradiction. Hence $d=\infty$. Assume $0<c$. Then $f(c)=0$ or $g(c)=0$. In this case $f(0) g(0)=0$. Because if $f(0) g(0)>0$, then $f(t)>0, g(t)>0$ for $t \in(0, \infty)$ by the preceding argument. Let

$$
A=B=\left(\begin{array}{cc}
\sqrt{2} c & 0 \\
0 & c
\end{array}\right), T=\frac{2 c}{\sqrt{3}}\left(\begin{array}{cc}
\frac{1}{2} & \frac{1}{2} \\
\frac{1}{2} & \frac{1}{2}
\end{array}\right) .
$$

Then $0 \leq A, B, T$ and $T^{2} \leq A^{2}=B^{2}$. Let $x=y=\left(\begin{array}{l}0 \\ 1\end{array}\right)$. Then

$$
\langle U f(|T|) g(|T|) x, y\rangle=\frac{1}{2} f\left(\frac{2 c}{\sqrt{3}}\right) g\left(\frac{2 c}{\sqrt{3}}\right) \neq 0
$$

and

$$
\|f(A) x\|\|g(B) y\|=\left\|\left(\begin{array}{c}
0 \\
f(c)
\end{array}\right)\right\|\left\|\left(\begin{array}{c}
0 \\
g(c)
\end{array}\right)\right\|=0 .
$$

This is a contradiction. Thus $c=0$ and $f, g$ are semi-operator monotone on $(0, \infty)$.

Next we show a direct extension of the Heinz-Kato inequality.

Theorem 6. Let $T \in B(\mathcal{H}), 0 \leq A, B \in B(\mathcal{H})$ and $\|T x\| \leq\|A x\|,\left\|T^{*} y\right\| \leq\|B y\|$ for $x, y \in \mathcal{H}$. Then, if a non-zero function $f$ is semi-operator monotone on $(0, \infty)$, then we have

$$
|\langle T x, y\rangle| \leq\|f(A) x\|\|g(B) y\|
$$

for $x, y \in \mathcal{H}$, where $g(t)=t / f(t)$.

Conversely, if continuous functions $f, g:[0, \infty) \rightarrow[0, \infty)$ satisfy conclusion (2) and $f(t) g(t)=t$ for $t \in(0, \infty)$, then $f, g$ are semi-operator monotone on $(0, \infty)$.

Proof. Let $f$ be semi-operator monotone on $(0, \infty)$. Then $\left\{f\left(t^{\frac{1}{2}}\right)\right\}^{2}$ is operator monotone on $(0, \infty)$ and $0<f\left(t^{\frac{1}{2}}\right)$ for $t \in(0, \infty)$. Hence $\left\{g\left(t^{\frac{1}{2}}\right)\right\}^{2}=t /\left\{f\left(t^{\frac{1}{2}}\right)\right\}^{2}$ is operator monotone on $(0, \infty)$ by [4, Corollary 2.6]. Thus $g$ is semi-operator monotone on $(0, \infty)$. Hence

$$
\begin{aligned}
|\langle T x, y\rangle| & =|\langle U|T| x, y\rangle| \\
& =|\langle U f(|T|) g(|T|) x, y\rangle| \\
& \leq\|f(A) x\|\|g(B) y\| \quad \text { for } x, y \in \mathcal{H}
\end{aligned}
$$

by Theorem 5 . The converse is easy from Theorem 5 .

Remark 7. For example, $f(t)=\sqrt{1+t^{2}}$ is semi-operator monotone on $(0, \infty)$. Hence, if $\|T x\| \leq\|A x\|,\left\|T^{*} y\right\| \leq\|B y\|$ for $x, y \in \mathcal{H}$, then

$$
|\langle T x, y\rangle| \leq\left\|\sqrt{1+A^{2}} x\right\|\left\|\frac{B}{\sqrt{1+B^{2}}} y\right\| \quad \text { for } x, y \in \mathcal{H} .
$$




\section{ACKNOWLEDGEMENTS}

We express sincere thanks to Professor M. Fujii, who kindly pointed out to us paper [2]. Also, we express sincere thanks to the referee for his helpful comments.

\section{REFERENCES}

[1] J. S. Aujla, Perturbation bounds for certain operator functions, Math. Inequal. Appl., 4 (2001), 609-617. MR 2002h:47015

[2] J. I. Fujii and M. Fujii, An analogue to Hansen's theory of generalized Löwner's functions, Math. Japonica, 35 (1990), 327-330. MR 91d:47015

[3] T. Furuta, An extension of the Heinz-Kato theorem, Proc. Amer. Math. Soc., 120 (1994), 785-787. MR 94e:47034

[4] E. Hansen and G. K. Pedersen, Jensen's inequality for operators and Löwer's theorem, Math. Ann., 258 (1982), 229-241.

[5] E. Heinz, Beiträge zur Störungstheorie der Spektralzerlegung, Math. Ann., 123 (1951), 415438. MR 13:471f

[6] T. Kato, Notes on some inequalities for linear operators, Math. Ann., 125 (1952), 208-212. MR 14:766e

[7] M. Uchiyama, Further extension of the Heinz-Kato-Furuta inequality, Proc. Amer. Math. Soc., 127 (1999), 2899-2904. MR 2000a:47045

Department of Mathematics, Tohoku Pharmaceutical University, Sendai 981-8558, JAPAN

E-mail address: tanahasi@tohoku-pharm.ac.jp

Mathematical Institute, Tohoku University, Sendai 980-8578, Japan

E-mail address: uchiyama@math.tohoku.ac.jp

Department of Mathematics, Fukuoka University of Education, Munakata 811-4192, JAPAN

E-mail address: uchiyama@fukuoka-edu.ac.jp 